\title{
2e Conférence Internationale sur le Paludisme et les Babésioses
}

\author{
19-22 septembre 1983/Annecy (France)
}

\section{2nd International Conference on Malaria and Babesiosis}

\author{
19-22 september 1983/Annecy (France)
}

“ La seconde Conférence Internationale sur le Paludisme et les Babésioses aura lieu à Annecy du 19 au 22 semptembre 1983. Cette réunion est placée sous l'égide de la Fondation Marcel Mérieux, de la Fondation Rockefeller, de l'Organisation Mondiale de la Santé, des Ministères français de la Recherche et de l'Industrie, de la Santé, de l'Agriculture, de la Coopération, de l'Agence Américaine pour le Développement International et du Centre de Recherche de l'Armée Américaine. Un programme préliminaire et des documents d'inscriptions sont disponibles en s'adressant au Dr M. Roumiantzeff, Fondation Mérieux, 17, rue Bourgelat, 69002 Lyon, France. Tél. (7) 838.06.10 poste 512 ».

Des Microfiches et des Microfilms de Cette pUblication peuvent Être obtenUS AUPRÈS DE: This publication is available in Microform from :

MASSON - SPPIF (réf. MIMC) I20, boulevard Saint-Germain, 75280 PARIS CEDEX 06

C) Masson, Paris, 1983 .

Le Directeur de la Publication: Dr J. TALAMon

Tous droits de traduction, d'adaptation et de reproduction par tous procédés pour tous pays

La loi du I I mars I957 n'autorisant, aux termes des alinéas 2 et 3 de l'article 4 I, d'une part. que les $\alpha$ copies ou reproductions strictement réservées à l'usage privé du copiste et non destinées à une utilisation collective » et, d'autre part, que les analyses et les courtes citations dans un but d'exemple et d'illustration, " toute représentation ou reproduction intégrale ou partielle, faite sans le consentement de l'auteur ou de ses ayants droit ou ayants cause, est illicite " (alinéa I ${ }^{\mathrm{er}}$ de l'article 40).

Cette représentation ou reproduction, par quelque procédé que ce soit, constituerait donc une contrefaçon sanctionnée par les articles 425 et suivants du Code pénal.

Masson, éditeur, Paris. - Dépôt légal : 1983. - No d’ordre : 5505. — mars 1983

IMPRIMERIE DARANTIERE DIJON-QUETIGNY (FRANCE). - - Commission paritaive $n^{\circ} 54169$

Printed in France. 